\title{
COMPARISON OF TWO FORECASTING METHODS IN TIME SERIES DATA WITH SEASONALITY
}

\author{
David Ramamonjisoa \\ Faculty of Software and Information Science, Iwate Prefectural University \\ 152-52 Sugo, Takizawa Iwate Japan
}

\begin{abstract}
This paper describes two forecasting methods in time series data with seasonality. The first method is an exponential smoothing model (parametric model) and the second forecast method is a machine learning model (artificial neural network model). We used a time series data with seasonality such as sunspot number data to evaluate the models. Our experiments show that the second forecast method has a better result in the sunspot data. We have also understood the difficulty in the modeling and implementation of those methods to forecasting and discuss their use in a real world application. Correlation of low season of sunspots and the low market prices is also observed.
\end{abstract}

\section{KEYWORDS}

Time Series Forecasting, Holt-Winters Method, LSTM Method, Sunspot Number Data

\section{INTRODUCTION}

There are two goals in handling a time series data. The first one is to make a descriptive analytic of the time series. The second one is to predict future events of the series. The descriptive analytic is the study of the data by exploring its structure and identifying patterns. A prediction goal is to forecast the possible outcome of the series in the future according to some models. The number of new coronavirus infected persons during the year 2020 is an example of a time series data. Any financial transaction data is another example.

This paper describes two forecasting methods in time series data with seasonality or cycle. The first method is an exponential smoothing model (parametric model) and the second forecast method is a machine learning model (artificial neural network model). We used a time series data with seasonality such as sunspot number data to evaluate the models. Our experiments show that the second forecast method has a better result in the sunspot data. We have also understood the difficulty in the modeling and implementation of those methods to forecasting and discuss their use in a real world application.

We first describe the time series problem, then we explain two forecasting models used in our experiments, we detail our experiment settings and finally we show our results and discussions on possible improvement and deployment.

\section{TIME SERIES PROBLEM AND ITS PROBLEM SOLVING}

Time series data is one of the most common formats of data, and it is used to describe an event or phenomena that occurs over time. Time series data has a unique important characteristic as one of the main attribute of the series is the time interval which the data is captured. Other common characteristics of time series data are seasonality, trend, cycles, and correlation.

The problem in time series data concerns with the process of extracting meaningful insights with the use of data visualization tools, statistical applications, and mathematical models to learn and explore past events and to forecast future events. There are four steps in the problem solving process such as data collection, data preparation, descriptive analysis and predictive analysis. 
- Data collection: Thanks to the exploding data publicly available on the internet, data can be easily collected and used for the problem we are dealing with. Academic institutions or international organizations have put online many raw data in order to be used and analyzed.

- Data preparation: Raw data need to be pre-processed such as filling or removing missing values, cleaning the unnecessary, and formatting to time series data. This is a process like in data science method on preprocessing.

- Descriptive analysis: In data science, this process is called the exploration data analysis (EDA). It is during this process that we identify the characteristics of the time series data. Summary statistics, patterns, distributions, cycles, and seasonality are examined. Time series are usually decomposed into the common characteristics basic time series. The trend, level, seasonality and white noise of the time series can be observed after the analysis.

- Predictive analysis: Forecasting models are built according to the time series characteristics. Statistical and mathematical models are the conventional method to forecast future events but machine learning models are also used to forecast time series data. In this paper, we focus on two forecasting models namely Holt-Winters and LSTM.

\section{TIME SERIES FORECASTING MODELS}

Two forecasting models are studied and implemented in this paper. We describe the mathematical functions of these models in the following.

\subsection{Holt-Winters Forecasting Method}

Time series data with seasonality are the main target of this model called Holt-Winters method according to their authors Holt and Winters (S. A. Roberts, 1982). Holt-Winters method is also called a triple exponential smoothing method. The basic formula is as follows in additive seasonality with noise or unpredictable values (Schmueli, G. and Lichtendahl, K.C., 2016):

Forecast $=$ estimated level + trend + seasonality at most recent time point

In mathematical notation we can write as in equation below:

$$
\mathrm{F}_{\mathrm{t}+\mathrm{h}}=L_{t}+h T_{t}+S_{t+h-M}
$$

Where level $\left(\mathrm{L}_{\mathrm{t}}\right)$, trend $\left(\mathrm{T}_{\mathrm{t}}\right)$, seasonality $\left(S_{t}\right)$ with season(M) and $h$ is the forecast horizon.

The forecast time series is then the addition of the three time series. The updating of those time series are executed according to the following formula depending on the three smoothing parameters $\alpha, \beta$, and $\gamma$.

Level: $\mathrm{L}_{\mathrm{t}}=\alpha \frac{Y_{t}}{S_{t-M}}+(1-\alpha)\left(L_{t-1}+T_{t-1}\right)$ or $\mathrm{L}_{\mathrm{t}}=\alpha\left(Y_{t}-S_{t-M}\right)+(1-\alpha)\left(L_{t-1}+T_{t-1}\right)$

Trend: $\mathrm{T}_{\mathrm{t}}=\beta\left(L_{t}-L_{t-1}\right)+(1-\beta) T_{t-1}$

Seasonality: $\mathrm{S}_{\mathrm{t}}=\gamma \frac{Y_{t}}{L_{t}}+(1-\gamma) S_{t-M}$ or $\mathrm{S}_{\mathrm{t}}=\gamma\left(Y_{t}-L_{t}\right)+(1-\gamma) S_{t-M}$

In terms of the observed data and forecasted data (predicted data), we can write as follow the equality $\hat{Y}_{t+h}=F_{t+h}$. The computation of this method is realized with the forecast package developed by Robert Hyndman (Hyndman R.J. and George Athanasopoulos, 2018) or stats package developed by David Meyer in $\mathrm{R}$ programming language.

\subsection{LSTM Model (Machine Learning Model)}

Thanks to the improvement of the computation power of personal computer, machine learning model can be easily executed within hours instead of several days of training for individual user. Users can also pay for cloud platform to obtain speed in computation and shorter execution time.

The mathematical model of machine learning algorithm such as LSTM (Long Short Term Memory) is briefly described as follows.

The time series data is basically the same as the sequence data in any form based on hypothesis where new input data depends on long sequence past data. Recurrent Neural Networks (RNNs) are first introduced to deal such a problem by using the output state as the next input of the node. The training of the RNNs model consists 
with finding the best weights on the network that the model fits best to all patterns in the training. But due to the RNNs architecture, the weight gradient computation is vanishing or exploding and long term dependency can't be learned. Hence, the neuron is modified to be a cell who can forget, input, output states or memories and the authors called it an LSTM (Sepp Hochreiter; Jürgen Schmidhuber (1997)). The RNN transformation cell equation is as below.

$$
\hat{y}_{t}=\operatorname{sigmoid}\left(\mathrm{W}_{\mathrm{y}} x_{t}+U_{y} c_{t-1}+b_{y}\right)
$$

And the LSTM transformation cell equations are as below.

$$
\begin{gathered}
\mathrm{f}_{t}=\operatorname{sigmoid}\left(\mathrm{W}_{\mathrm{f}} x_{t}+U_{f} c_{t-1}+b_{f}\right) \\
\mathrm{i}_{t}=\operatorname{sigmoid}\left(\mathrm{W}_{\mathrm{i}} x_{t}+U_{i} c_{t-1}+b_{i}\right) \\
\mathrm{o}_{t}=\operatorname{sigmoid}\left(\mathrm{W}_{\mathrm{o}} x_{t}+U_{o} c_{t-1}+b_{o}\right) \\
\mathrm{c}_{t}=\mathrm{f}_{\mathrm{t}} \circ \mathrm{c}_{\mathrm{t}-1}+\mathrm{i}_{\mathrm{t}} \circ \tanh \left(\mathrm{W}_{\mathrm{c}} x_{t}+b_{c}\right) \\
h_{t}=\mathrm{o}_{\mathrm{t}} \circ \tanh \left(\mathrm{c}_{\mathrm{t}}\right)
\end{gathered}
$$

Where $f_{t}$ is the forget gate, $i_{t}$ is the input gate, $o_{t}$ is the output gate, $c_{t}$ is the cell, and $h_{t}$ is the state.

The implementation of the LSTM is realized with tensorflow and keras modules (Cholet, F. and Allaire, J.J. 2018) in R programming language.

\section{TIME SERIES FORECASTING EXPERIMENTS}

In this section, we describe the experimentation and forecasting results for the two models. We used the monthly sunspot number data that we have gathered from the sunspot index and long-term solar observations based in Brussels recognized as the International Sunspot Number (ISN) (Solar Influences Data analysis Center (SIDC) http:// http://www.sidc.be/silso/). The variation in the sunspot activity is referred as "solar cycle". Cycle length can vary from 9 to 12 years. There is also a magnetic cycle of 22 years where the magnetic polarity of the sun flips from one solar cycle to one another. The peak of the sunspot counts in each cycle is called the solar cycle maximum and the trough is the solar cycle minimum. Those peaks and troughs vary according to the cycle and at some point at the beginning in 1645 to 1715 there are zero or few sunspots for a period of 60 years. This period is called a Maunder Minimum. The "Little Ice Age" occurred over parts of Earth during the Maunder Minimum. The cycle is interpreted as two seasons. High sunspot season have high magnetic field energy which can be measured with the indicator $10.7 \mathrm{~cm}$ solar radio flux. This indicator is also a time series data that measures have started in 1950s and it correlates 100 percent with the ISN. There are currently 24 solar cycles in the dataset and soon or later we are going to observe the beginning of the solar cycle 25 . The time series predictive analytic summary is presented as in the table 1.

Table 1. ISN time series data summary

\begin{tabular}{ll}
\hline Time Series Data Provider & Solar Influences Data analysis Center (SIDC) \\
\hline Name & International Sunspot Number (ISN) called also $R$ \\
Time Interval & Monthly \\
Time Start, Time End & 1749-01, 2019-12 \\
Statistical summary & Min:0, Mean:51,Max:253, length:3252 \\
Characteristics & Seasonality=Yes, type: additive, cycle=132 $\mp 40$ months (11years $\mp$ 1.2years), \\
Properties & Univariate time series \\
\hline
\end{tabular}

The forecasting results obtained from the two models are depicted the figure 1. The data is split into train and test data. We use the $92 / 8$ as a percentage ratio for the split. The HoltWinters method requires optimized parameters in order to forecast well. The default parameters aren't the optimized values. Through a good iniatilization and grid search for 100 million steps, we have obtained the results in the figure 1 . More optimization grid search is necessary to achieve meaningful results. The LSTM setting is very simple as we put 200 LSTM cells in the input layer and a dense layer with one output to predict the next value. The training uses a batch during the backpropagation and stops at a certain accuracy with no improvement in the loss function results. 


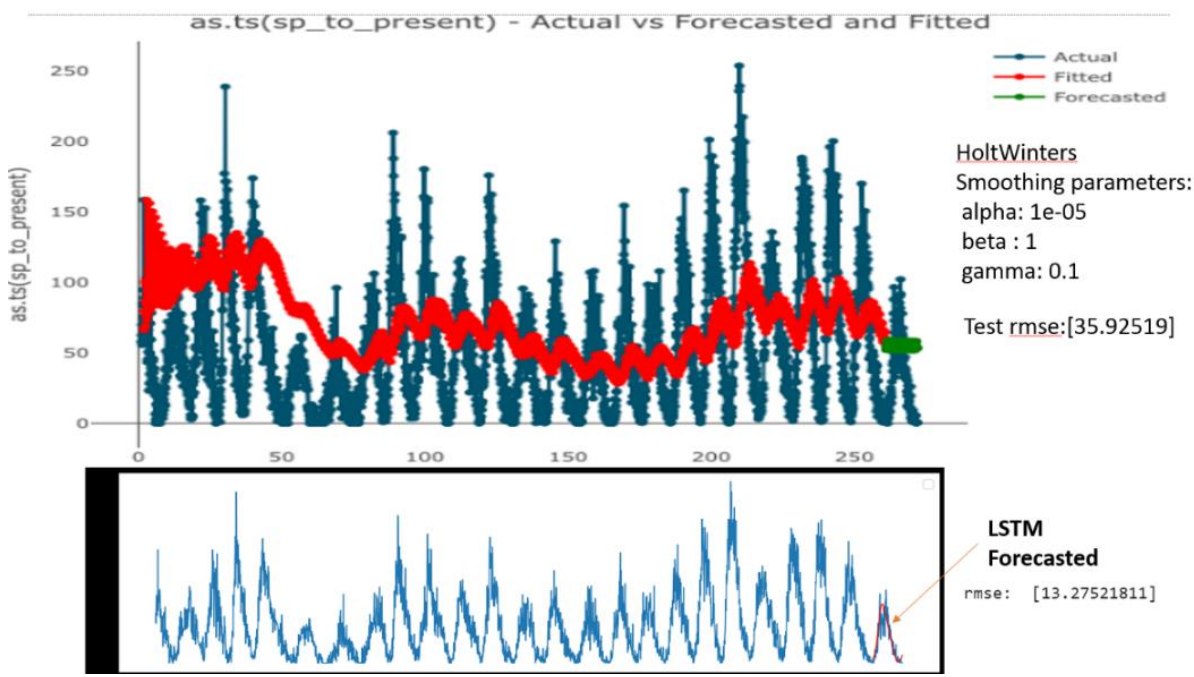

Figure 1. The actual time series sunspot number data and the results of the forecasting by HoltWinters method and LSTM model

\section{CONCLUSION}

Time series forecasting is part of the applied computing and a classical application in data science.

We presented two forecasting model techniques for time series data with seasonality. The first one is a triple exponential smoothing method called Holt-Winters model and the second one is a machine learning model called LSTM. We used the sunspot number data to evaluate the models. We observed that the Holt-Winters model doesn't perform well during the forecasting nor correctly fit the training data. We optimized the smoothing parameters with grid search to make a better model. The LSTM model is a blackbox that an incremental tuning on the learning parameters and training size must be performed iteratively in order to obtain some interesting results. Autonomous machine learning are now becoming an active research area but many problems yet to solve. We are planning to extend our experiment to those new techniques.

The sunspot data is an active research problem in time series analysis. The nature of the Sun and physics law allow to understand the plasma, magnetic field sunspots, dynamo and their cyclical phenomena but it is not clear how it can predict the next cycle pattern or the pattern in 2050 for example without time series forecasting.

In the future, we will apply the models to financial real world time series data as we have been working before (Ramamonjisoa, D. and Yoshiki Sato, 2017) by finding correlations such as seasonal period and market trends. The low season of sunspots has an important impact in living system bigger than we know.

\section{REFERENCES}

Cholet, F. and Allaire, J.J. 2018. Deep Learning with R. Manning Publications Co, New York, USA.

Hyndman R.J. and George Athanasopoulos, 2018. Forecasting Principles and Practice. OTexts Publishing Co., USA.

Ramamonjisoa, D. and Yoshiki Sato, 2017. Analysis of the Nikkei 225 Index and Future Prediction. Proceedings of the 10th IADIS Information Systems. Budapest, Hungary, pp. 175-179.

S. A. Roberts, 1982. A General Class of Holt-Winters Type Forecasting Models. In Management Science, Vol. 28, No. 7 , pp 808-820. https://doi.org/10.1287/mnsc.28.7.808

Schmueli, G. and Lichtendahl, K.C., 2016. Practical Time Series Forecasting with R. Axelrod Schnall Publishers, USA.

Sepp Hochreiter; Jürgen Schmidhuber (1997). Long short-term memory. In Neural Computation. 9 (8): 1735-1780. doi:10.1162/neco.1997.9.8.1735. PMID 9377276. 